\title{
Profilaxis antimicrobiana previa a procedimientos dentales. Situación actual y nuevas perspectivas
}

\author{
Rodríguez-Campos LF', Ceballos-Hernández $\mathrm{H}^{2}$, Bobadilla-Aguirre $\mathrm{A}^{3}$
}

\section{Resumen}

La bacteremia secundaria a los procedimientos dentales puede dar origen a episodios de endocarditis infecciosa. La implementación de la profilaxis antimicrobiana tiene como finalidad aminorar la bacteremia transitoria reduciendo la posibilidad de desarrollar endocarditis infecciosa, por lo que es indispensable conocer qué antibióticos son de elección y en qué casos o situaciones administrarlos u omitirlos. Existen múltiples guías para aplicar profilaxis antimicrobiana, éstas difieren en sus recomendaciones, a tal grado que pueden confundir al facultativo al momento de determinar si el paciente requiere antibiótico profiláctico y a elegir el antimicrobiano adecuado.

OBJETIVO: comparar las diferentes guías de profilaxis antimicrobiana y recomendaciones para converger en una recopilación única con las aportaciones destacadas de cada guía revisada; de la misma manera, exponer el régimen utilizado en nuestra institución por el servicio de estomatología.

CONCLUSIÓN: el estomatólogo se puede basar principalmente en las guías de la American Heart Association (AHA) y el Centro Nacional de Excelencia Tecnológica en Salud (CENETEC) para condiciones cardiacas que ameriten profilaxis antimicrobiana, y para pacientes con enfermedades sistémicas sobre la guía de la American Academy of Pediatric Dentistry (AAPD) y las recomendaciones de nuestra institución. La administración de profilaxis antimicrobiana se da por razones científicas consolidadas y por carácter de tipo médico-legal, en un futuro se espera establecer criterios para aumentar los métodos no antibióticos preventivos de endocarditis infecciosa y aminorar el uso de antimicrobianos, reduciendo el uso de antibiótico profiláctico, sin exponer al paciente a infecciones diseminadas y con mínimos efectos secundarios posibles.

PALABRAS CLAVE: profilaxis antibiótica, endocarditis, inmunosupresión, recomendaciones.

\footnotetext{
${ }^{1}$ Ex-Residente de Estomatología Pediátrica. ${ }^{2}$ Adscrito del Servicio de Estomatología Pediátrica. 3Jefe del Servicio de Cardiología Pediátrica.
}

Instituto Nacional de Pediatría, Ciudad de México.

Recibido: 5 de mayo del 2017

Aceptado: 28 de julio del 2017

\section{Correspondencia}

Hilda Ceballos-Hernández hilda.ceballoshdz@gmail.com Luis Fernando Rodríguez-Campos

mcd.luisfernando@gmail.com

\section{Este artículo debe citarse como}

Rodríguez-Campos LF, Ceballos-Hernández H, BobadillaAguirre A. Profilaxis antimicrobiana previa a procedimientos dentales. Situación actual y nuevas perspectivas. Acta Pediatr Mex. 2017;38(5):337-350. 
Acta Pediatr Mex. 2017 Sep;38(5):337-350.

\title{
Antimicrobial prophylaxis prior to dental procedures. Current situation and new perspectives.
}

\author{
Rodríguez-Campos LF', Ceballos-Hernández $\mathrm{H}^{2}$, Bobadilla-Aguirre $\mathrm{A}^{3}$
}

\begin{abstract}
Secondary bacteremia from dental procedures can produce episodes of infective endocarditis (IE), the implementation of antimicrobial prophylaxis (AP) aims to reduce transient bacteremia reducing the possibility of developing IE. So it is essential to know which antibiotics are of choice and in which cases to administer them or in which situations we can omit them. Currently there are multiple guidelines for applying AP, these guidelines differ on their recommendations to such a degree that they can confuse the practitioner in determining if the patient requires prophylactic antibiotic and choose the appropriate antimicrobial.
\end{abstract}

OBJECTIVE: To compare different guidelines and recommendations in order to converge on a compilation with the outstanding contributions of each guide reviewed, in the same way expose the current regime used at the stomatology service at our Institution.

CONCLUSIONS: The stomatologist decisions can be based mainly on the American Heart Association (AHA) and Centro Nacional de Excelencia Tecnológica en Salud (CENETEC) guidelines for cardiac conditions where antimicrobial prophylaxis is warranted, and for patients with systemic diseases on the American Academy of Pediatric Dentistry (AAPD) guideline and the recommendations of our institution. Therefore in the future establish criteria to increase the use of non antibiotics methods to prevent infectious endocarditis and minimize the use of antimicrobial agents reducing the use of prophylactic antibiotic without exposing the patient to disseminated infections with minimal side effects.

KEYWORDS: antibiotic prophylaxis; endocarditis; immunosuppression; recommendations
${ }^{1}$ Ex-Residente de Estomatología Pediátrica. ${ }^{2}$ Adscrito del Servicio de Estomatología Pediátrica.

${ }^{3}$ Jefe del Servicio de Cardiología Pediátrica.

Instituto Nacional de Pediatría, Ciudad de México.

\section{Correspondence}

Hilda Ceballos-Hernández hilda.ceballoshdz@gmail.com Luis Fernando Rodríguez-Campos mcd.luisfernando@gmail.com

\section{INTRODUCCIÓN}

Desde hace más de 50 años, la diseminación hematógena de bacterias de la cavidad oral se ha considerado un factor decisivo en la patogénesis del $10 \%$ al $15 \%$ de los episodios de endocarditis infecciosa, lo que sugiere que ciertos procedimientos dentales pueden representar un factor de riesgo significativo. ${ }^{1-4}$

La idea de que muchas infecciones sistémicas podrían producirse por infecciones de la cavidad 
oral y que el tratamiento dental conservador podría favorecer este proceso, tuvo especial importancia a principios del siglo XX. En 1900, Hunter escribió: "Los empastes de oro, coronas y puentes construidos en y alrededor de las raíces de los dientes enfermos forman un verdadero mausoleo sobre una masa de sepsis para el que no hay paralelo en todo el ámbito de la medicina o cirugía" ${ }^{1,5}$ Desde estas décadas se ha señalado a la práctica estomatológica como fuente potencial de ciertas infecciones que pudieran derivar de procedimientos bucales que al generar sangrado, permiten la introducción al torrente sanguíneo de los microorganismos específicos, sus productos metabólicos y mediadores de la inflamación, causando efectos sistémicos o contribuir a enfermedades multisistémicas, tal es el caso de la endocarditis infecciosa, infecciones de prótesis articulares, abscesos extracardiacos (esplénicos, vertebrales, cerebrales o renales), condiciones sistémicas como el síndrome de inmunodeficiencia humana, inmunodeficiencias primarias o secundarias, leucemias, desnutrición, diabetes mal controlada, las cuales, por el estado físico desfavorable de la persona, podrían favorecer el desarrollo de infecciones producidas por microorganismos que en un organismo inmunológicamente competente serían inocuos.

Con la finalidad de evitar o reducir en lo posible algunas de estas situaciones se prescribe de manera preventiva antibiótico anticipado a procedimientos dentales, no sólo enfocado a la previsión de endocarditis infecciosa, sino también evitar en lo posible infecciones a distancia, ampliando así, el contexto de profilaxis antibiótica a profilaxis antimicrobiana. ${ }^{3,4,6-11}$

El uso de los antimicrobianos está indicado en pacientes odontológicos en diversas situaciones clínicas para evitar que, debido a los procedimientos realizados, se expongan a ciertos microorganismos patógenos que podrían constituir un riesgo importante de infección focal, causada por un foco de infección primario que se irradia a partes distales o sistémicas.

Dentro los principales microorganismos encontramos S. viridans, este microorganismo, habitante frecuente en bocas sanas, forma parte de $30 \%$ de la flora del surco gingival causante de cerca del $50 \%$ del total de endocarditis infecciosa, de los cuales el $1 \%$ son causados por procedimientos dentales. ${ }^{1,3,4,6,12-15}$

Actualmente, contamos con múltiples guías para la aplicación de profilaxis antimicrobiana (American Heart Association, National Institute for Health and Care Excellence, European Society of Cardiology), entre éstas, existen algunas discrepancias con relevancia a la aplicación de la profilaxis antimicrobiana (americana, europea, británica), lo que es fácil de comprender si tenemos en cuenta que no hay una evidencia absoluta afianzada en muchos aspectos de su implementación. ${ }^{16,17}$ La unificación de criterios hará más fácil para el facultativo suministrar u omitir oportunamente la profilaxis antimicrobiana.

\section{OBJETIVO}

El objetivo de esta revisión es comparar las diferentes guías y recomendaciones acerca de profilaxis antimicrobiana para converger en una recopilación única con las aportaciones destacadas de cada guía revisada; de la misma manera, exponer el régimen utilizado en nuestra institución por el servicio de estomatología en la actualidad.

\section{JUSTIFICACIÓN DE LA PROFILAXIS ANTIMICROBIANA}

Se ha evidenciado que la magnitud de las bacteremias transitorias resultantes del tratamiento dental se considera baja y similar a la que se presenta por realizar actividades cotidianas como: cepillarse los dientes, uso de hilo dental, 
o el masticar alimentos. Es muy plausible especular que el riesgo infeccioso de las actividades cotidianas de higiene dental presentan un riesgo superior al de las intervenciones odontológicas como: extracciones, cirugía periodontal, raspado y alisado radicular y procedimientos endodónticos ${ }^{1,3,11-14,18,19}$ (Cuadro 1). De hecho, es 1000 a 8000 veces más probable que una bacteremia sea provocada por manipulaciones orales diarias que por procedimientos dentales. El fracaso en

Cuadro 1. Frecuencia de bacteremias temporales inducidas por tratamientos y autoinducidas

\begin{tabular}{|c|c|c|}
\hline Procedimiento dental & $\begin{array}{c}\text { Frecuencia de } \\
\text { bacteremias }\end{array}$ & Referencias \\
\hline $\begin{array}{l}\text { Inyecciones } \\
\text { intraligamentarias de } \\
\text { anestesia en niños }\end{array}$ & 16 a $97 \%$ & 46 \\
\hline Extracciones dentales & 10 a $46 \%$ & 29 \\
\hline Cirugía periodontal & 36 a $88 \%$ & 19 \\
\hline Endodoncia & 8 a $80 \%$ & 19 \\
\hline Detartraje ultrasónico & $53 \%$ & 44 \\
\hline Sondeo periodontal & $43 \%$ & 14 \\
\hline Profilaxis & 0 a $40 \%$ & 19 \\
\hline $\begin{array}{l}\text { Colocación de banda } \\
\text { matriz con cuña }\end{array}$ & $32 \%$ & 45 \\
\hline Irrigación subgingival & $30 \%$ & 31 \\
\hline $\begin{array}{l}\text { Colocación de grapa } \\
\text { para dique de hule }\end{array}$ & $29 \%$ & 45 \\
\hline Pulido dental & $24 \%$ & 45 \\
\hline Remoción de suturas & 11 a $16 \%$ & 8 \\
\hline \multicolumn{3}{|l|}{$\begin{array}{l}\text { Actividades orales de } \\
\text { rutina diaria }\end{array}$} \\
\hline Uso de hilo dental & 0 a $58 \%$ & 19 \\
\hline Masticación & 17 a $51 \%$ & 19 \\
\hline $\begin{array}{l}\text { Dispositivo irrigador } \\
\text { de agua }\end{array}$ & 7 a $50 \%$ & 19 \\
\hline Enjuague bucal & $50 \%$ & 20 \\
\hline Palillos de dientes & 20 a $40 \%$ & 9 \\
\hline Cepillado dental & 0 a $26 \%$ & 19 \\
\hline
\end{tabular}

Tomado de: Skaug N. Bakken V. Complicaciones Sistémicas de las infecciones endodóncicas. Bergenholtz G, HorstedBindslev P, Reit C. Endodoncia. 2da Ed. El Manual Moderno. 2011:128-139. la administración de profilaxis antimicrobiana apropiada puede generar acusaciones por negligencia médica. ${ }^{4,10,12,13,19-21}$

Según Baltch (1988), la bacteremia que se observa 5 minutos después de la inducción bacteriana presenta una incidencia bacteriémica de 58 a $76 \%$ sin uso de antibióticos, en comparación con 14 a $16 \%$ con la profilaxis con antibióticos, y a los 30 minutos una incidencia de 26 a $51 \%$ frente al 3-9\% reportados por Hall (1993), de tal manera, que la bacteremia transitoria post procedimiento no se elimina completamente por los antibióticos. . $20,22^{2}$

Hasta ahora no hay datos que demuestren que reducir la duración o la frecuencia de la bacteremia después de cualquier procedimiento médico conlleve menos riesgo de endocarditis infecciosa relacionada con la intervención. De manera similar, no hay evidencia suficiente de estudios que respalde la necesidad de la profilaxis de la endocarditis infecciosa. Sin omitir lo anterior, la aplicación de profilaxis antimicrobiana se justifica en razones científicas-médicas consolidadas y por orden médico-legal justificando que el consenso general tienda a aplicar la profilaxis, pero actualmente ya disponemos de recomendaciones para prescindir de su empleo..$^{1,3,11-14,18,19}$

Por lo general, las bacteremias son asintomáticas y temporales (duración menos de 15 a 30 min) debido a que el número de células bacterianas en la sangre es bajo $(<10$ unidades formadoras de colonias por $\mathrm{mL}$ ). Además, el sistema reticuloendotelial y la respuesta inmune humoral de huésped eliminan rápidamente los microorganismos. Por tanto, en individuos saludables, las bacterias generalmente no tienen importancia clínica y son asintomáticas; sin embargo, en individuos que carecen de protección normal contra las infecciones (pacientes comprometidos) las bacterias pueden empezar a multiplicarse en la sangre, dando como resultado una sepsis local 
o generalizada, que viene acompañada de manifestaciones sistémicas de inflamación (fiebre, dolor, malestar general, aumento de proteína C reactiva, etc.). En huéspedes comprometidos (p. ej. pacientes con cáncer, diabetes no controlada o inmunodeficiencias) la bacteremia puede progresar a una infección general letal.,10.12,13,19-21

En el Cuadro 2 se muestra las guías de las principales asociaciones y sociedades médicas en relación a las recomendaciones para la administración de profilaxis antimicrobiana, 3,13,23-27 así como los criterios utilizados en nuestra institución.

Koulocheris y sus colaboradores sugieren que la profilaxis antimicrobiana durante los procedimientos quirúrgicos orales y maxilofaciales debe llevarse a cabo durante al menos seis meses después de la finalización de la quimioterapia; misma recomendación se aplica para pacientes con terapia inmunosupresora. ${ }^{28,29}$ En el Cuadro 3 se describen los procedimientos odontológicos que ameritan profilaxis antimicrobiana. La profilaxis con antibióticos pueden inducir resistencia a múltiples antibióticos en los patógenos graves, alergia, toxicidad y repercusión gastrointestinal (colitis), esto, combinado con los costos financieros, requiere criterios estrictos para una relación riesgo-beneficio aceptable. 17,20,30

La American Heart Association (AHA) no recomienda la administración de profilaxis antimicrobiana en procedimientos de operatoria dental, tallado protésico y colocación de provisionales, tratamiento de conductos en los que no se rebase la unión cemento-dentinaria, colocación de postes, toma de radiografías, remoción de puntos de sutura, colocación, ajuste o remoción de aditamentos de ortodoncia, colocación de puentes removibles, exfoliación de dientes deciduos o traumatismo de los labios y la mucosa bucal; sin embargo, la administración de profilaxis antimicrobiana es imperativa siempre que se prevea sangrado. ${ }^{3,6,14}$

\section{REGÍMENES DE PROFILAXIS ANTIMICRO- BIANA RECOMENDADOS}

Las diferentes guías manejan un régimen antibiótico similar; pero los esquemas profilácticos recomendados por la American Heart Association (AHA) son los más utilizados y se acercan más a los referidos por el Centro Nacional de Excelencia Tecnológica en Salud (CENETEC), privilegian la vía oral, lo cual facilitan su administración y cumplimiento, por lo que los demás regímenes antimicrobianos de las distintas directrices de la European Society of Cardiology (ESC) y British Society for Antimicrobial Chemotherapy (BSAC) escapan a esta revisión. El antimicrobiano de elección debe ser administrado en un momento tal que proporcione las concentraciones séricas y tisulares superiores a la concentración inhibitoria mínima para los organismos asociados con el procedimiento.

En el Cuadro 4 se exponen los esquemas profilácticos. ${ }^{3,4,6,14}$ La profilaxis antimicrobiana en todas las modalidades debe administrarse una hora antes del procedimiento dental que implique riesgo y, si por alguna razón involuntaria el paciente no cumplió con las indicaciones, podría administrarse en el consultorio inmediatamente antes del procedimiento o sólo en este caso extremo, hasta 2 h después del mismo.

Sólo en aquellos casos en los que la semivida del antibiótico es inferior a una hora y la duración de la intervención es de 2-3 horas, o más de dos veces la semivida del antibiótico, sería necesario repetir dosis. De forma similar si se produjese una pérdida sanguínea superior a 1-2 L durante el procedimiento debería considerarse la administración de una dosis adicional de antibiótico, sobre todo en procedimientos de cirugía maxilofacial. ${ }^{6,31-33}$

Como ya se mencionó en el Cuadro 4 la amoxicilina es el antibiótico de primera opción a utilizar 


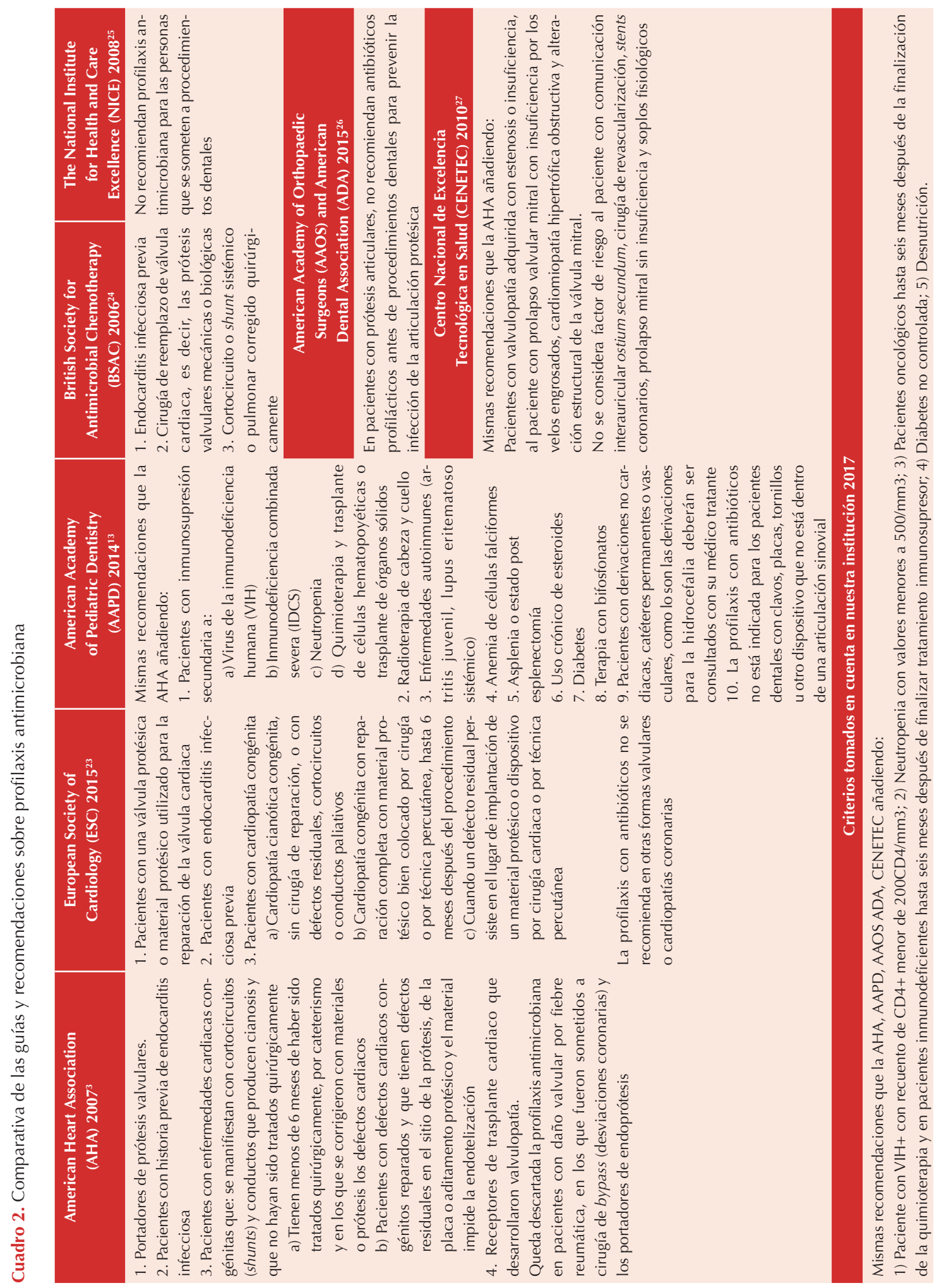


Cuadro 3. Procedimientos bucales que requieren profilaxis antibiótica. Prevención de endocarditis infecciosa; guía de la American Heart Association 2007*

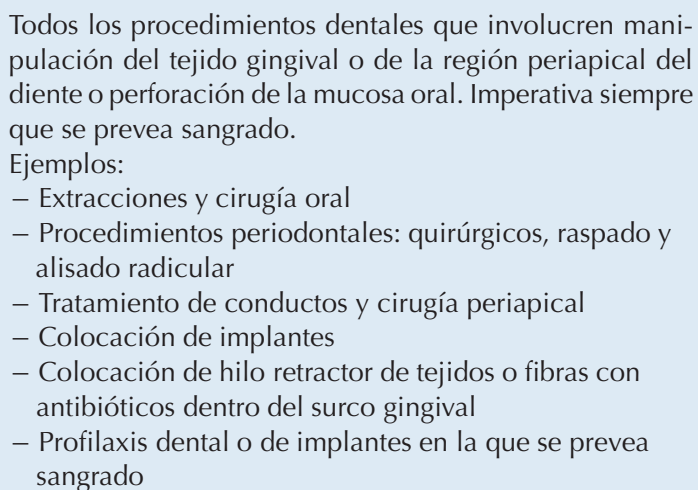

*Modificado de: Díaz L, Castellanos J. Prevención de endocarditis infecciosa en Odontología. Nuevas recomendaciones sobre profilaxis antibiótica (2007). REV ADM 2007;54(4);126-130.

Cuadro 4. Régimen de profilaxis antimicrobiana en procedimientos dentales AHA $2007^{3,4,6,14}$

\begin{tabular}{|c|c|c|c|}
\hline \multirow{2}{*}{$\begin{array}{l}\text { Situación: tipo } \\
\text { de profilaxis }\end{array}$} & \multirow[t]{2}{*}{ Antibiótico } & \multicolumn{2}{|c|}{$\begin{array}{l}\text { Dosis } 30 \text { a } 60 \text { min antes } \\
\text { del procedimiento }\end{array}$} \\
\hline & & Adultos & Niños \\
\hline $\begin{array}{l}\text { Vía oral } \\
\text { estándar }\end{array}$ & Amoxicilina & $2 \mathrm{~g}$ VO & $50 \mathrm{mg} / \mathrm{kg}$ \\
\hline \multirow{3}{*}{$\begin{array}{l}\text { Alergia a la } \\
\text { penicilina }\end{array}$} & Clindamicina & $600 \mathrm{mg}$ VO & $20 \mathrm{mg} / \mathrm{kg}$ \\
\hline & Cefalexina* & $2 \mathrm{~g} \mathrm{VO}$ & 50 mg/kg \\
\hline & $\begin{array}{l}\text { Azitromicina o } \\
\text { claritromicina }\end{array}$ & $500 \mathrm{mg}$ VO & $15 \mathrm{mg} / \mathrm{kg}$ \\
\hline $\begin{array}{l}\text { Incapacidad } \\
\text { de administra- } \\
\text { ción oral }\end{array}$ & Ampicilina & 2 g IM o IV & 50 mg/kg \\
\hline \multirow{2}{*}{$\begin{array}{l}\text { Alergia a } \\
\text { penicilina e } \\
\text { incapacidad } \\
\text { de ingesta oral }\end{array}$} & $\begin{array}{l}\text { Cefazolina o } \\
\text { ceftriaxona* }\end{array}$ & $1 \mathrm{~g} \mathrm{IM} \mathrm{o} \mathrm{IV}$ & $50 \mathrm{mg} / \mathrm{kg}$ \\
\hline & Clindamicina & $\begin{array}{l}600 \mathrm{mg} \text { IM } \\
\text { o IV }\end{array}$ & $20 \mathrm{mg} / \mathrm{kg}$ \\
\hline
\end{tabular}

VO: vía oral; IM: intramuscular; IV: intravenoso. La dosis total en niños no deberá superar la dosis de adultos.

*No utilizar cefalosporinas si existen antecedentes de anafilaxia, angioedema o urticaria con penicilina.

en la profilaxis antimicrobiana. La clindamicina es la principal alternativa cuando no es plausible administrar amoxicilina (riesgo de anafilaxia); asimismo, la clindamicina es de elección en pacientes inmunodeprimidos o inmunosuprimidos por ser un antimicrobiano de amplio espectro con actividad contra los aerobios grampositivos y una extensa gama de bacterias anaerobias, entre ellas los patógenos productores de betalactamasa, además reduce la virulencia de las bacterias y refuerza las actividades fagocíticas de los linfocitos inmunitarios del huésped, convirtiéndolo idóneo en estos pacientes, pero siempre vigilando el riesgo de colitis asociada a antibióticos y como segunda opción en pacientes con cardiopatía alérgicos a la penicilina. ${ }^{34-36}$ Por otra parte, existen métodos no antibióticos para la prevención de endocarditis infecciosa (Cuadro 5). ${ }^{37}$

En un estudio realizado por Barbosa y sus colaboradores en 2015, donde se realizaron enjuagues previos con clorhexidina $0,2 \%(10 \mathrm{~mL}$ durante 1 minuto), se evidenció que al realizar extracción dental con anestesia local se reduce significativamente la duración (en 15 minutos) de bacteremia secundaria, hasta un 34\% menos.

El objetivo principal de la profilaxis con antisépticos locales es reducir la carga bacteriana

Cuadro 5. Prevención de endocarditis infecciosa. Manejo con métodos no antibióticos

1. Controlar estado gingivoperiodontal antes de progra mar cualquier procedimiento de tipo electivo.

2. Controlar cualquier estomatitis viral o micótica antes de programar algún procedimiento de tipo electivo.

3. Evitar o reducir manipulaciones prolongadas o trau máticas.

4. Optimizar tiempo operatorio.

5. Prescribir uso de enjuagues antisépticos ligeros, para uso cotidiano.

6. Usar enjuagues o aplicaciones directas de clorhexidina al $0.2 \%$ antes de cada sesión ( $10 \mathrm{~mL}$ durante un minuto).

Tomado y modificado de: Castellanos JL, Díaz-Guzmán L, Gay-Zárate O. Medicina en odontología. Manejo dental de pacientes con enfermedades sistémicas. 2da Edición. Manual Moderno 2002. México.45-54. 
presente en la cavidad oral en el momento de iniciar la manipulación, con el fin de minimizar el riesgo de desarrollo de una bacteremia. Esto confirma la idoneidad de la realización de un enjuague bucal con clorhexidina al 0,2\% antes de extracciones de dientes, con el fin de reducir la duración de bacteremia postextracción. ${ }^{2}$ Esta práctica quizá debería extenderse a todas las manipulaciones dentales, así como otras medidas preventivas con métodos no antibióticos ya planteadas.

Dayer y sus colaboradores reportan en el 2015 un aumento en la incidencia de endocarditis infecciosa utilizando la guía del National Institute for Health and Care Excellence (NICE) en Inglaterra, en un periodo tres años, donde exponen que el riesgo potencial de prescindir de profilaxis antibiótica resulta en un extra de 419 casos de endocarditis infecciosa por año, incluyendo 66 posibles muertes adicionales. En contraste, el riesgo potencial de aplicación de las directrices de la European Society of Cardiology para dar profilaxis antibiótica podría resultar en un extra de siete reacciones adversas a los medicamentos notificables a un año, incluyendo una muerte cada 3 años (Figura 1). ${ }^{25,38,39}$

\section{¿CUÁL GUÍA SEGUIR?}

Al no contar con una Norma Oficial Mexicana acerca de profilaxis antimicrobiana, sólo tenemos la guía CENETEC (Centro Nacional de Excelencia Tecnológica en Salud) como referencia; sin embargo, aunque cuenta con criterios similares a la AHA (American Heart Association) no profundiza sobre los procedimientos que requieren profilaxis antimicrobiana y no incluye enfermedades sistémicas. Las guías de la AHA, European Society of Cardiology (ESC), British Society for Antimicrobial Chemotherapy (BSAC) y CENETEC en sus últimas ediciones tienen directrices similares y se enfocan en patologías cardiacas. La guía American Academy of Ortho- paedic Surgeons American Dental Association (AAOS-ADA) ya no recomienda administrar profilaxis antimicrobiana en pacientes con prótesis articulares y la guía de la Academy of Pediatric Dentistry (AAPD) cuenta con las mismas recomendaciones que la $\mathrm{AHA}$ en cuanto a cardiopatías pero añade pacientes con enfermedades que ocasionen compromiso inmunológico ya sea de carácter autoinmune, hematooncológico o de inmunosupresión causada por esteroides. Por lo tanto, de las guías revisadas, a nuestra consideración ninguna engloba todos los criterios necesarios, siendo la de la AAPD la más completa ya que abarca condiciones cardiacas como sistémicas. Descartamos el uso de la guía del National Institute for Health and Care Excellence (NICE) ya que recomienda no aplicar profilaxis antimicrobiana y esto podría resultar en un aumento de endocarditis infecciosa o de infecciones metafocales. En nuestra institución utilizamos las mismas recomendaciones que la AHA, AAPD, AAOS-ADA y CENETEC añadiendo los criterios enumerados en el Cuadro 2, prescindimos de pautas de las guías ESC y BSAC por su similitud con la AHA.

La decisión de administrar o no antibiótico profiláctico es del estomatólogo, y a manera de contestar la pregunta: ¿qué guía seguir?, si nos enfocamos en condiciones cardiacas podemos optar por las guías AHA, ESC, BSAC y CENETEC, con especial énfasis en AHA y CENETEC; para pacientes con enfermedades sistémicas la guía AAPD y las recomendaciones de nuestra institución.

\section{DISCUSIÓN}

En ocasiones, por la alta demanda en nuestra institución de atención estomatológica, pacientes foráneos, niños que serán intervenidos quirúrgicamente del corazón o ingresarán a terapia antineoplásica o inmunosupresora que requieren rehabilitación bucal prioritaria urgente antes de 


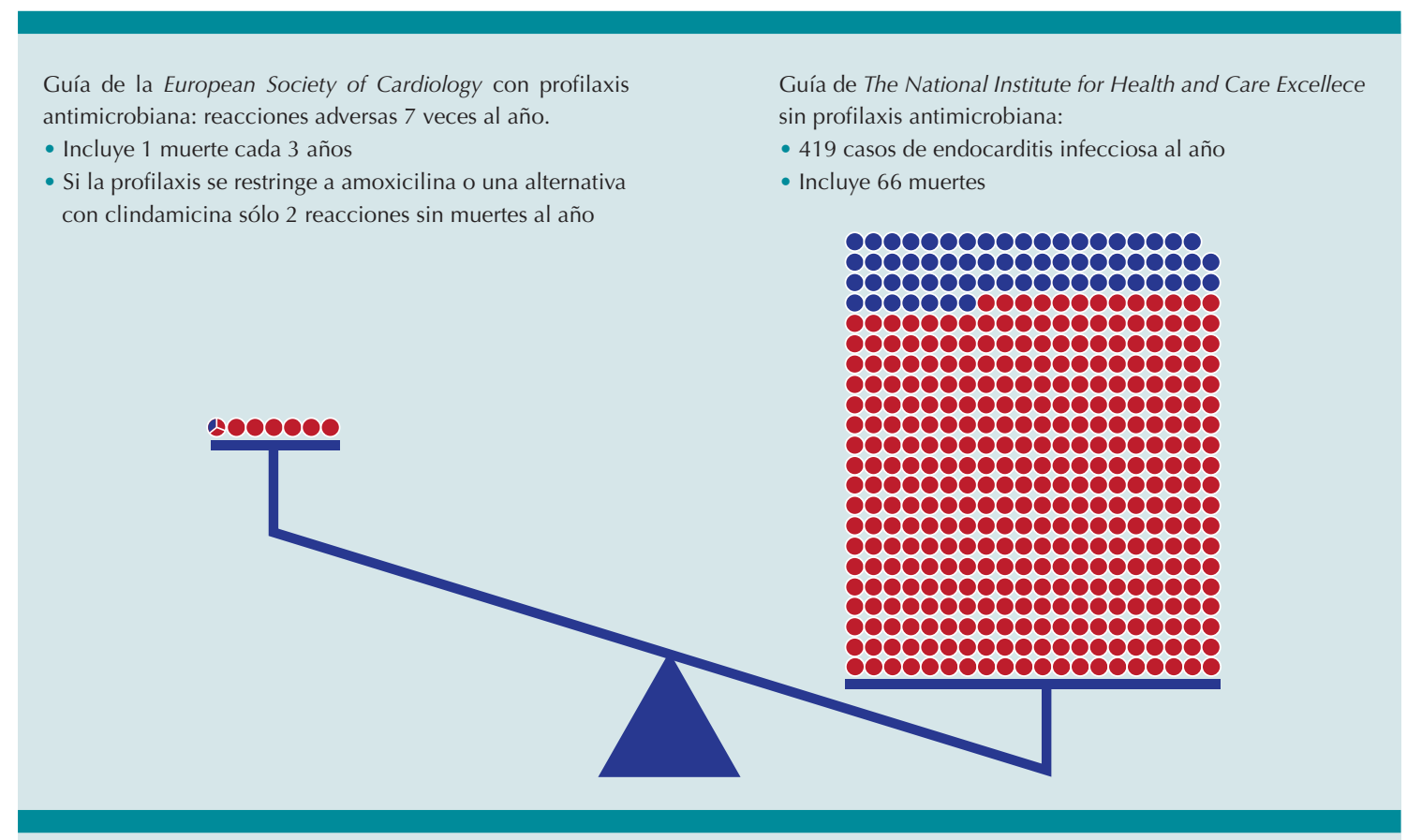

Figura 1. Los riesgos de recomendar la profilaxis antimicrobiana (PA) vs. sin PA. Modificado de: M.H. Thornhill, M. Dayer, P. B. Lockhart, M. McGurk, D. Shanson. Guidelines on prophylaxis to prevent infective endocarditis. British Dental Journal: 2016;220:51-56.

comenzar con su tratamiento, es necesario realizar 2 procedimientos dentales o más por día, con la finalidad de dejarlos libres de procesos infecciosos en cavidad bucal en el menor tiempo posible; en este tipo de situaciones se puede aplicar claritromicina o azitromicina, ya que se sustentan en el suero más prolongadamente que la amoxicilina y clindamicina, ${ }^{40}$ dicho régimen es aplicable en pacientes foráneos para atenderlos dos veces por día (mañana y tarde), así como cuando no es posible obtener clindamicina en suspensión, ya que en nuestra experiencia hemos observado que la clindamicina en suspensión tiene un costo elevado y cada vez es más difícil de adquirir. En pacientes hospitalizados que serán rehabilitados en un lapso comprendido de 3 a 5 días, se puede optar por dosis terapéuticas de antibiótico durante la rehabilitación terminando el esquema del antibiótico elegido, disminuyendo así el desarrollo de resistencia bacteriana. En el Cuadro 6 se puntualizan las principales enfermedades en niños en las que se administra profilaxis antimicrobiana en nuestra institución.

Es primordial comprender cuándo se requiere de profilaxis antimicrobiana y cuándo es necesario establecer el antibiótico de forma terapéutica convencional, sobre todo en los casos donde ya existe infección latente (abscesos odontogénicos o periodontales) se sustituye la profilaxis antimicrobiana por la impregnación de antibiótico y se cumple el esquema según el fármaco a utilizar, esto también se aplica en pacientes que serán rehabilitados en lapsos cortos y continuos menores a una semana, donde se prefiere utilizar esquema antibiótico a profilaxis antimicrobiana, así como en pacientes con traumatismos dentoalveolares donde recomendamos impregnar al paciente 
Cuadro 6. Principales padecimientos pediátricos a los que se les administra profilaxis antimicrobiana en nuestra institución

\begin{tabular}{|c|c|c|c|}
\hline Cardiacos & Inmunológicos & Hemato-oncológicos & Nefrológicos \\
\hline $\begin{array}{l}\text { Pacientes diagnosticados con } \\
\text { lo siguiente: } \\
\text { Cardiopatías acianóticas o de } \\
\text { cianosis tardía: } \\
\text { - Comunicación interventri- } \\
\text { cular (CIV) } \\
\text { - Persistencia del conducto } \\
\text { arterioso (PCA) } \\
\text { - Estenosis pulmonar } \\
\text { - Coartación de la aorta } \\
\text { Todas las cardiópatas congéni- } \\
\text { tas cianóticas: } \\
\text { - Tetralogía de Fallot } \\
\text { - Anomalía de Eibstein } \\
\text { - Atresia pulmonar } \\
\text { - Atresia tricuspídea } \\
\text { - Transposición de grandes } \\
\text { vasos }\end{array}$ & $\begin{array}{l}\text { Pacientes con enfermedades que } \\
\text { cursen con inmunodepresión } \\
\text { o inmunosupresión (esteroides, } \\
\text { antimaláricos, metrotexate) } \\
\text { - Lupus eritematoso sistémico } \\
\text { - Dermatomiositis } \\
\text { - Púrpura de Henoch-Schönlein } \\
\text { - Artritis idiopática juvenil } \\
\text { - Púrpura trombocitopénica } \\
\text { idiopática } \\
\text { - Síndrome antifosfolípido } \\
\text { - Enfermedad de Kawasaki } \\
\text { - Encefalitis autoinmune } \\
\text { (Anti-NMDA) } \\
\text { - Infecciosa-inmunodeficiencia: } \\
\text { VIH+ con CD4 menor a 200, } \\
\text { carga viral mayor a } 3000 \text {, } \\
\text { neutropenia menor a } 500\end{array}$ & $\begin{array}{l}\text { Cualquier paciente con } \\
\text { cáncer que se encuentre } \\
\text { con terapia antineoplásica } \\
\text { - Leucemias, principal } \\
\text { mente linfoblástica } \\
\text { aguda } \\
\text { - Osteosarcomas } \\
\text { Histiocitosis de células } \\
\text { de Langerhans } \\
\text { - Neuroblastoma } \\
\text { - Retinoblastoma } \\
\text { - Sarcomas } \\
\text { - Linfomas } \\
\text { - Tumor de Wilms } \\
\text { - Rabdomiosarcoma } \\
\text { - Anemia aplásica } \\
\text { Pos-trasplantados de } \\
\text { células madres hemato- } \\
\text { poyéticas en tratamiento } \\
\text { inmunosupresor }\end{array}$ & $\begin{array}{l}\text { Cualquier paciente con } \\
\text { nefropatía en tratamien- } \\
\text { to inmunosupresor } \\
\text { - Enfermedad renal } \\
\text { crónica } \\
\text { - Síndrome nefrótico } \\
\text { - Síndrome nefrítico } \\
\text { - Paciente en diálisis } \\
\text { Pos-trasplantados } \\
\text { renales en tratamiento } \\
\text { inmunosupresor } \\
\text { Evitar en estos pacien- } \\
\text { tes uso de antibióticos } \\
\text { con alta nefrotoxicidad } \\
\text { (tetraciclinas) }\end{array}$ \\
\hline
\end{tabular}

con antibiótico, realizar el procedimiento, ya sea reimplantación de dientes, ferulización, sutura de tejidos blandos, etc., y posteriormente continuar con el régimen antibiótico; de esta manera el antibiótico es coadyuvante junto con la eliminación local del proceso infeccioso de origen dental y previene la endocarditis bacteriana en pacientes de riesgo, evitando la diseminación de infecciones a distancia en pacientes inmunocomprometidos.

Lo anterior se basa en que, excepto en los pacientes con un sistema inmune comprometido, los antibióticos no son curativos, pero en cambio funcionan para ayudar en el restablecimiento de un equilibrio adecuado entre las defensas del huésped (inmunes e inflamatorias) y el(los) agente(s) invasivo(s).

Muy rara vez los antibióticos son sustitutos adecuados para la eliminación de la fuente de infección (extracción, tratamiento endodóntico, incisión y drenaje, raspado y alisado radicular). En casos donde la infección sea demasiado difusa o diseminada para identificar un nido para la incisión o la situación clínica no permita el tratamiento curativo inmediato, el estomatólogo prudentemente decidirá administrar terapia antibiótica hasta el momento en que el tratamiento curativo puede ser implementado. Cuando la causa es fácilmente identificable es imprescindible eliminar el foco de la infección previo a, o concomitante con la terapia antibiótica. ${ }^{41}$

En contraparte, en un panorama global, el índice de dientes cariados, perdidos, obturados (CPOD) es una determinante a nivel mundial para conocer la epidemiologia de la caries (Cuadro 7). Países europeos como Reino Unido, Alemania, Dinamarca y Suiza presentan un valor menor a 0.5 de CPOD a comparación de países del continente americano que presentan un índice promedio de 2.4 como es el caso de México, así como alrededor de 1.5 para Estados Unidos 
Rodríguez-Campos LF et al. Profilaxis antimicrobiana en procedimientos dentales

Cuadro 7. Procedimientos que requieren profilaxis antimicrobiana vs. procedimientos que requieren antibioticoterapia convencional

¿Cuándo administrar profilaxis antimicrobiana?

Se implementará en todos los procedimientos dentales que involucren manipulación del tejido gingival o de la región periapical del diente o perforación de la mucosa oral. Imperativa siempre que se prevea sangrado.

Ejemplos:

- Infiltración de anestesia local, por sí sola no representa alto riesgo pero la anestesia en la mayoría de los casos va de la mano de procedimientos invasivos

- Procedimientos de operatoria dental que involucren sangrado por colocación de grapa y dique de hule

- Procedimientos de cirugía bucal y maxi lofacial

- Procedimientos periodontales y endo dónticos

- Preparación para coronas en dientes per manentes donde se prevea sangrado

- Preparación para coronas de acero inoxidable pediátricas donde se provoque sangrado

- Colocación de hilo retractor
¿Cuándo administrar antibiótico con régimen de esquema completo?

1) Sí ya existe infección latente se optara por la impregnación antibiótica por lo menos $30 \mathrm{~min}$ antes del procedimiento y continuar con la terapéutica antibiótica convencional

2) En traumatismos dentoalveolares que ameriten manipulación de tejidos duros o blandos

Ejemplos:

1) Abscesos odontogénicos

Abscesos periodontales

Infección de heridas quirúrgicas

2) Reimplante de dientes avulsionados Sutura de laceraciones de mucosa oral: lengua, mucosa yugal, frenillos
No se recomienda profilaxis antimicrobiana

- Operatoria dental con grapas atrau máticas para colocación de selladores o fisurotomía

- Preparación para prótesis fija inlay y onlay.

- Colocación de provisionales

- Tratamiento de conductos en los que no se rebase la unión cementodentinaria $^{6}$

- Colocación de endopostes

- Toma de radiografías

- Toma de impresiones

- Remoción de puntos de sutura

- Colocación, ajuste o remoción de aditamentos de ortodoncia

- Colocación de prótesis removibles

- Exfoliación de dientes temporales

- Colocación de fluoruro de América. De tal razón es más frecuente la administración de profilaxis antimicrobiana en países con un alto CPOD, ya que presentan mayor incidencia de caries $y$, por lo tanto, tienden más a ser sometidos a procedimientos dentales. Por lo cual, el origen preventivo para evitar en lo posible la administración antibiótica profiláctica se encuentra en prever la aparición de caries dental, sobre todo en pacientes de alto riesgo (cardiópatas, inmunodeprimidos e inmunosuprimidos). ${ }^{42}$

\section{CONCLUSIÓN}

La administración de profilaxis antimicrobiana se da por razones científicas consolidadas y por carácter de tipo médico-legal tomando en cuenta el siguiente decálogo:
1. Recordar que el dentista es responsable del tratamiento dental del paciente y la decisión de aplicar o prescindir de antibiótico profiláctico se basará en obtener los mejores intereses del paciente (riesgobeneficio) y evitar reacciones adversas: alérgicas, resistencia antimicrobiana, interacción con medicamentos de base, hipersensibilidad y en casos extremos, síndrome de Stevens-Johnson. ${ }^{6}$

2. La finalidad de la profilaxis antimicrobiana es prevenir en lo posible el desarrollo de endocarditis infecciosa, así como la proliferación y diseminación bacteriana que originen una infección a distancia en pacientes no inmunocompetentes.

3. El dogma central consiste en indicar profilaxis antimicrobiana siempre que exista un 
riesgo importante de infección, propiciado por la condición local y sistémica del paciente (cardiopatías congénitas, inmunodepresión) y las características propias del procedimiento (ineludible siempre que se prevea sangrado).

4. Se debe motivar constantemente al paciente a mantener una adecuada salud periodontal y de la misma manera a controles periódicos de placa dentobacteriana e implementar los métodos no antibióticos de prevención de endocarditis.

5. Realizar enjuague bucal con clorhexidina 0,2\% o como mínimo al $0,12 \%$ a razón de $10 \mathrm{~mL}$ durante 1 minuto antes de cualquier manipulación dental, sobre todo en aquellas que impliquen sangrando como: extracciones, curetajes, incisión de tejidos blandos.

6. Diferenciar los casos que requieren profilaxis antimicrobiana de los que requieren impregnación y terapia antibiótica continua y de igual manera los procedimientos que lo ameritan, lo mencionado se describe en el Cuadro 7.

7. El antimicrobiano de elección debe ser seguro y con espectro bacteriano apto, en nuestra institución, el servicio de estomatología utiliza amoxicilina como primera opción en pacientes cardiópatas, ${ }^{3,12}$ clindamicina para pacientes inmunosuprimidos por la gama bacteriana a destruir y azitromicina para pacientes que serán atendidos más de una vez el mismo día por la alta vida media de este fármaco.

8. En pacientes con enfermedad renal avanzada se optará por antibióticos de eliminación hepática.
9. Dirigir las estrategias preventivas con enfoque a disminuir el índice de dientes cariados, perdidos, obturados de la población mexicana.

10. Como recopilación y recomendación final, el estomatólogo se puede basar en las guías AHA, ESC, BSAC y CENETEC para condiciones cardiacas que ameriten profilaxis antimicrobiana, con especial énfasis en AHA y en la Guía de Práctica Clínica de la CENETEC acerca de Diagnóstico y Tratamiento de la Endocarditis Infecciosa; para pacientes con enfermedades sistémicas, sobre la guía AAPD y las recomendaciones de nuestra institución.

Los criterios sobre los que se apoya el servicio de estomatología en nuestra institución para administrar profilaxis antimicrobiana se basan en los recomendados por la AHA, AAPD, AAOS-ADA, los anexados en el Cuadro 2 y las recomendaciones de la CENETEC. El alto éxito en la prevención de endocarditis infecciosa e infecciones metafocales (abscesos cerebrales, esplénicos, renales, infecciones oportunistas) se fundamenta en la adecuada implementación de la profilaxis antimicrobiana.

\section{NUEVAS PERSPECTIVAS}

Desde la introducción de la primera recomendación de profilaxis antibiótica por la AHA (American Heart Association) en los años 50, se ha dado un giro completo en sus directrices haciéndolas mucho más sencillas de seguir. Por tal motivo, en un futuro es imprescindible aumentar los métodos no antibióticos preventivos de endocarditis infecciosa y disminuir el uso de antibióticos en base a recomendaciones y guías con un contexto reducido en cuanto al procedimiento y al tipo de paciente candidato a profilaxis antimicrobiana, aminorando el uso de antibiótico profiláctico sin exponer al paciente a infecciones diseminadas. 
Rodríguez-Campos LF et al. Profilaxis antimicrobiana en procedimientos dentales

\section{REFERENCIAS}

1. Tomás I, Álvarez M. Pathogenesis of endocarditis: Bacteraemia of oral origin. In: Breijo-Márquez FR, editor. Endocarditis. InTech; 2012:19-50.

2. Barbosa M, Prada-López I, Álvarez M, Amaral B, de los Angeles Casares-De-Cal M, Tomás I. Post-Tooth Extraction Bacteraemia: A Randomized Clinical Trial on the Efficacy of Chlorhexidine Prophylaxis. PLoS ONE. 2015;10(5):1-15.

3. Wilson W, Taubert KA, Gevitz M. Prevention of in-fective endocarditis: Guidelines from the American Heart Association-A Guideline From the American Heart Association Rheumatic Fever, Endocarditis and Kawasaki Disease Committee, Council on Cardiovascular Disease in the Young, and the Council on Clinical Cardiology, Council on Cardiovascular Surgery and Anesthesia Anes-thesia, and the Quality of Care and Outcomes Research Interdisciplinary Working Group. J Am Dent Assoc 2007;138(6):739-45, 747-60. Erratum in: J Am Dent Assoc 2008;139(3):253.

4. Little J. Falace D, Miller C. Dental Management of the Medically Compromised Patient. Elsevier, 8th Edition, 2013. Chapter 18: AIDS, HIV Infection, and Related Conditions; 293-301.

5. Hunter W. Oral sepsis as a cause of disease. British Medical Journal, 1900;2065(2):215-216.

6. Lee EA, Profilaxis antimicrobiana. Castellanos JL, Díaz- Guzmán L, Gay- Zárate O. Medicina en odontología. Manejo dental de pacientes con enfermedades sistémicas. 3ra Edición. Manual Moderno 2014. México: 237-250.

7. Pizzo G, Guiglia R, Lo Russo L, Campisi G. Dentistry and internal medicine: from the focal infection theory to the periodontal medicine concept. Eur J Intern Med. 2010;21:496-502.

8. Asensi V, Alvarez M, Carton JA, Lago M, Maradona JA, Asensi JM. Eikenellacorrodens brain abscess after repeated periodontal manipulations cured with imipenem and neurosurgery. Infection. 2002;30:240-242.

9. Brady P, Bergin S, Cryan B, Flanagan O. Intracranial abscess secondary to dental infection. J Ir Dent Assoc. 2014;60:32-34.

10. Skaug N. Bakken V. Complicaciones Sistémicas de las infecciones endodóncicas. Bergenholtz G, Horsted-Bindslev P, Reit C. Endodoncia. 2da Ed. El Manual Moderno. 2011:128-139.

11. Habib G, Hoen B, Tornos P, Thuny F, Prendergast B, Vilacosta I. Guía de práctica clínica para prevención, diagnóstico y tratamiento de la endocarditis infecciosa. Grupo de Trabajo de la Sociedad Europea de Cardiología (ESC) para prevención, diagnóstico y tratamiento de la endocarditis infecciosa en colaboración con la European Society of Clinical Microbiology and Infectious Diseases (ESCMID) y la International Society of Chemotherapy (ISC) forInfection and Cancer. Rev Esp Cardiol. 2009; 62(12):1465.e1-.e54.

12. Lockhart PB, Brennan MT, Kent ML, Norton HJ, Weinrib DA. Impact of amoxicillin prophylaxis on the incidence, nature, and duration of bacteremia in children after intubation and dental procedures. Circulation. 2004;109:2878-84.

13. American Academy On Pediatric Dentistry Clinical Affairs Committee, Guideline on antibiotic prophylaxis for dental patients at risk for infection. Pediatricdentistry, 2008;30(7):215. Revised 2014. Disponible en: http:// www.aapd.org/media/policies_guidelines/g_antibioticprophylaxis.pdf

14. Díaz L, Castellanos JL: Prevención de endocarditis infecciosa en Odontología. Nuevas recomendaciones sobre profilaxis antibiótica. Rev ADM. 2007;54(4);126-130.

15. Baltimore RS, Gewitz M, Baddour LM. Beerman LB. Jackson MA. Lockhart PB. Willoughby R. Infective Endocarditis in Childhood: 2015 Update. Circulation, 2015;132(15):14871515.

16. Sánchez A. Delgado-Iribarren A. Recomendaciones para la prevención de endocarditis: a quien, cúando y cómo. Inf Ter Sist Nac Salud. 2009;33:39-48.

17. Gopalakrishnan PP, Shukla SK, Tak T. Infective Endocarditis: Rationale for Revised Guidelines for Antibiotic Prophylaxis. Clinical Medicine \& Research. 2009;7(3):63-68.

18. Forner L, Larsen T, Kilian M, Holmstrup P. Incidence of bacteremia after chewing, tooth brushing and scaling in individuals with periodontal inflammation. J Clin Periodontol. 2006;33:401-407.

19. Roberts GJ. Dentists are innocent! "Everyday" bacteremia is the real culprit: a review and assessment of the evidence that dental surgical procedures are a principal cause of bacterial endocarditis in children. Pediatr Cardiol. 1999;20:317-325.

20. Pallasch TJ, Slots J. Antibiotic prophylaxis and the medically compromised patient. Periodontol 2000. 1996;10:107-138.

21. Badran Z, Struillou X, Verner C, Clee T, Rakic M, Martinez $M C$, Soueidan A. Periodontitis as a risk factor for systemic disease: Are microparticles the missing link? MedHypotheses. 2015;84(6):555-556.

22. Maharaj B, Coovadia Y, Vayej AC. A comparative study of amoxicillin, clindamycin and chlorhexidine in the prevention of post-extraction bacteraemia. Cardiovascular Journal of Africa. 2012;23(9):491-494.

23. Habib G, Lancelotti P, Antunes MJ. 2015 ESC Guidelines for the management of infective endocarditis: The Task Force for the management of Infective Endocarditis of the European Society of Cardiology (ESC): Endorsed by: European Association for Cardio-Thoracic Surgery (EACTS), the European Association of Nuclear Medicine (EANM). Eur Heart J 2015; p: ehv319.

24. Gould FK, Elliott TS, Foweraker J, Fulford M, Perry JD, Roberts GJ, and others. Guidelines for the prevention of endocarditis: report of the Working Party of the British Society for Antimicrobial Chemotherapy. J Antimicrob Chemother 2006;57(6):1035-42.

25. National Institute for Health and Care Excellence (NICE). Prophylaxisagainstinfective endocarditis. 2015. Disponible en: http://www.nice.org.uk/guidance/indevelopment/gidcgwave0748/documents (acceso agosto 2016). 
26. Sollecito TP, Abt E, Lockhart, PB, Truelove E, Paumier TM, Tracy SL, Frantsve-Hawley J. The use of prophylactic antibiotics prior to dental procedures in patients with prosthetic joints: evidence-based clinical practice guideline for dental practitioners - a report of the American Dental Association Council on Scientific Affairs. The Journal of the American Dental Association, 2015;146:11-16.

27. Guía de referenciarápida. Diagnóstico y Tratamiento de la Endocarditis Infecciosa. Guía de Práctica Clínica: IMSS404-10.Disponible en: http://www.cenetec.salud.gob.mx/ descargas/gpc/CatalogoMaestro/404_IMSS_10_Endocarditis_Infecciosa/GRR_IMSS_404_10.pdf

28. Koulocheris $P$, Metzger MC, Kesting MR, Hohlweg-Majert B. Life-threatening complications associated with acute monocytic leukaemia after dental treatment, Australian Dental Journal. 2009;54(1):45-48.

29. Zimmermann C, Meurer MI, Grando LJ. Dental treatment in patients with leukemia. J Oncol. 2015;1:1-14.

30. Stone H H, Haney B B, Kolb L D. Prophylactic and preventive antibiotic therapy: timing, duration, and economics. Ann Surg. 1979;189:691-699.

31. Gutiérrez JL, Bagán JV, Bascones A, Llamas R, Llena J, Morales A, Noguerol B, Planells P, Prieto J, Salmerón JI. Consensus document on the use of antibiotic prophylaxis in dental surgery and procedures. Med Oral Patol Oral Cir Bucal. 2006;11:188-205.

32. García-Rodríguez JA, Prieto J, Gobernado M.Gomis M, Mensa J. Documento de consenso sobre quimioprofilaxis quirúrgica. Rev Esp Quimioterap. 2000;13:205-13.

33. Nichols RL. Current approaches to antibiotic prophylaxis in surgery. Inf Dis Clin Pract. 1993;2:149-157.

34. Brook I, Lewis MAO, Sándor GKB, Jeffcoat M, Samaranayake LP, Vera-Rojas J. Clindamicina para el tratamiento de infecciones dentales. Revista ADM 2007;64(6):230-237.
35. Dar-Odeh NS, Abu-Hammad OA, Al-Omiri MK, Khraisat AS, Shehabi AA. Antibiotic prescribing practices by dentists: a review. Therapeutics and Clinical Risk Management. 2010;6:301-306.

36. Rodríguez-Alonso E. Rodríguez-Monje MT. Tratamiento antibiótico de la infección odontogénica. Inf Ter Sist Nac Salud. 2009;33:67-79.

37. Castellanos JL, Díaz- Guzmán L, Gay- Zárate O. Medicina en odontología. Manejo dental de pacientes con enfermedades sistémicas. 2da Edición. Manual Moderno 2002. México.45-54.

38. Dayer $M$ J, Jones $S$, Prendergast $B$, Baddour $L$ M, Lockhart $\mathrm{P} \mathrm{B}$, Thornhill $\mathrm{M} \mathrm{H}$. Incidence of infective endocarditis in England, 2000-2013: a secular trend, interrupted timeseries analysis. Lancet 2015;385:1219-1228.

39. Thornhill MH, Dayer M, Lockhart PB, McGurk M, Shanson D. Guidelines on prophylaxis to prevent infective endocarditis. British Dental Journal. 2016;220:51-56.

40. Vermot D, Entenza JM, Vouillamoz J, Glauser MP, Moreillon P. Efficacy of Clarithromycin versus That of Clindamycin for Single-Dose Prophylaxis of Experimental Streptococcal Endocarditis. Antimicrob Agents Chemother. 1996;40(3):809-11.

41. Morrow SG. Use and abuse of antibiotics. Endodontics: Colleagues for Excellence. Winter 2012. Disponible en: http:// www.aae.org/uploadedfiles/publications_and_research/ endodontics_colleagues_for_excellence_newsletter/ ecfewinter12final.pdf. Consultado Octubre 4, 2016.

42. Silveira R. Epidemiology of Dental Caries in the World, Oral Health Care-Pediatric, Research, Epidemiology and Clinical Practices. InTech:2012, Disponible en: http:// www.intechopen.com/books/oral-health-care-pediatricresearch-epidemiology-andclinical-practices/epidemiology-of-dental-caries-in-the-world 